\title{
Unveiling the long non-coding RNA profile of porcine reproductive and respiratory syndrome virus-infected porcine alveolar macrophages
}

Junxin Gao, Yu Pan, Yunfei Xu, Wenli Zhang, Lin Zhang, Xi Li, Zhijun Tian, Hongyan Chen and Yue Wang* (D)

\begin{abstract}
Background: Long noncoding RNA (IncRNA) is highly associated with inflammatory response and virus-induced interferon production. By far the majority of studies have focused on the immune-related IncRNAs of mice and humans, but the function of IncRNAs in porcine immune cells are poorly understood. Porcine reproductive and respiratory syndrome virus (PRRSV) impairs local immune responses in the lungs of nursery and growing pigs, whereas the virus triggers the inflammatory responses. Porcine alveolar macrophage (PAM) is the primary target cell of PRRSV, thus PRRSV is used as an in vitro model of inflammation. Here, we profiled IncRNA and mRNA repertories from PRRSV-infected PAMs to explore the underlying mechanism of porcine IncRNAs in regulating host immune responses.
\end{abstract}

Results: In this study, a total of 350 annotated IncRNAs and 1792 novel IncRNAs in PAMs were identified through RNA-seq analysis. Among them 86 differentially expressed (DE) IncRNAs and 406 DE protein-coding mRNAs were identified upon PRRSV incubation. GO category and KEGG pathway enrichment analyses revealed that these DE IncRNAs and mRNAs were mainly involved in inflammation- and pathogen infection-induced pathways. The results of dynamic correlated expression networks between IncRNAs and their predicted target genes uncovered that numerous IncRNAs, such as XLOC-022175, XLOC-019295, and XLOC-017089, were correlated with innate immune genes. Further analysis validated that these three IncRNAs were positively correlated with their predicted target genes including CXCL2, IFI6, and CD163. This study suggests that porcine IncRNAs affect immune responses against PRRSV infection through regulating their target genes in PAMs.

Conclusion: This study provides both transcriptomic and epigenetic status of porcine macrophages. In response to PRRSV infection, comprehensive DE IncRNAs and mRNAs were profiled from PAMs. Co-expression analysis demonstrated that IncRNAs are emerging as the important modulators of immune gene activities through their critical influence upon PRRSV infection in porcine macrophages.

Keywords: Porcine reproductive and respiratory syndrome virus, Long non-coding RNA, Porcine alveolar macrophage, mRNA-IncRNA correlation network

\footnotetext{
* Correspondence: wangyue@caas.cn

State Key Laboratory of Veterinary Biotechnology, Heilongjiang Provincial Key Laboratory of Laboratory Animal and Comparative Medicine, Harbin Veterinary Research Institute, Chinese Academy of Agricultural Sciences, Harbin, China
}

(c) The Author(s). 2021 Open Access This article is licensed under a Creative Commons Attribution 4.0 International License, which permits use, sharing, adaptation, distribution and reproduction in any medium or format, as long as you give appropriate credit to the original author(s) and the source, provide a link to the Creative Commons licence, and indicate if changes were made. The images or other third party material in this article are included in the article's Creative Commons licence, unless indicated otherwise in a credit line to the material. If material is not included in the article's Creative Commons licence and your intended use is not permitted by statutory regulation or exceeds the permitted use, you will need to obtain permission directly from the copyright holder. To view a copy of this licence, visit http://creativecommons.org/licenses/by/4.0/. The Creative Commons Public Domain Dedication waiver (http://creativecommons.org/publicdomain/zero/1.0/) applies to the data made available in this article, unless otherwise stated in a credit line to the data. 


\section{Background}

Next-generation sequencing of host genomes has revealed that non-coding RNAs, including micro RNAs, lncRNAs, and circular RNAs, are transcribed from most genomes [1]. LncRNAs, defined as non-coding RNAs of more than 200 nucleotides in length, are most often endowed with polyadenylation, positioned in the nucleus, cytoplasm or both [2,3]. Emerging evidence has shown that lncRNAs are involved in a variety of biological processes including genomic imprinting, cell proliferation and differentiation, and cellular developmental processes [4]. Transcriptome-wide studies have demonstrated that many lncRNAs exhibit highly tissue and cell type specific expression $[5,6]$, indicating that lncRNAs may be the driver of cell-specific response [7, 8]. For example, a terminal differentiation-induced lncRNA TINCR is specifically expressed in the late stage of human somatic tissue, which regulates the epidermal differentiation through TINCR-mRNA base-pairing interactions [9]. Several lncRNAs are tumor-specific, such as PVT1 [10] and HAGLROS [11], regulating the expression of oncogenes and tumor suppressor genes. LncRNA LOC646329, a cell proliferation regulator, appears low in neocortical tissues but enriches in radial glia cells [12]. These data indicated that lncRNAs may play important roles in lineage-specific differentiation or specialized cellular function.

The innate immune system is the first line of host defense against invading organisms. Recently lncRNAs have been identified as the key regulators in the innate immune response. One well-characterized lncRNA lincRNA-Cox2 has been identified to be a regulator of the transcription of macrophages, which can either activate the expression of IL- 6 and IL-23a via the TLR2 pathway or inhibit the expression of IFN-stimulated genes (IRF7, ISG15, IFI204, and OAS2) [13]. Another immune-related lncRNA THRIL is highly induced in monocyte cell line THP-1, serving as an enhancer of TNF $\alpha$ and IL-6 through interacting with hnRNPL [14]. However, little is known regarding the function of lncRNAs in porcine innate immune cells during virus infection.

Porcine reproductive and respiratory syndrome virus (PRRSV) is the etiologic agent of porcine reproductive and respiratory syndrome, which is characterized by respiratory problem in growing pigs and reproductive failure in sows [15, 16]. Porcine alveolar macrophages (PAMs) are the primary target cells of PRRSV. Macrophages, as a group of innate immune cells, play a central role in monitoring viral infections including PRRSV, influenza A virus, and HIV. To successfully fight against PRRSV infection, macrophages have evolved various strategies to regulate antiviral responses, such as regulating the production of IFN- $\alpha / \beta$ [17]. The previous evidence suggested that PRRSV was susceptible to IFNs [18], whereas PRRSV-mediated suppression of IFNs production helped the virus circumvent the host antiviral responses [19]. Besides, the pro-inflammatory cytokines including IL-1, IL-6, IL-8, and TNF- $\alpha$ mainly produced in PAMs after virus invasion, play critical roles in infection and pathogenesis of PRRSV [20-23]. However, the intracellular regulatory mechanisms of lncRNAs related to these innate immune properties remain to be addressed. Here, we used PRRSV as an inflammatory model to stimulate PAMs and explored the regulatory role of porcine lncRNAs in innate immune responses.

\section{Results}

\section{Expression profile of IncRNA in PAMs}

To evaluate the performance of endogenous lncRNAs in PAMs, we designed a synthetic reference pool from three specific-pathogen-free (SPF) Landrace pigs by RNA-seq analysis. More specifically, our pool contained 2142 lncRNAs (350 known lncRNAs and 1792 novel lncRNAs) and 22,565 mRNAs. To predict the function of these lncRNAs, we performed KEGG pathway analysis of cis- and trans-regulated predicted mRNAs. The result showed that enriched GO and KEGG pathways were mainly related to the inflammatory response, such as "MAPK signaling pathway", "cytokine-cytokine receptor interaction", "TNF signaling pathway", "toll-like receptor signaling pathway", and "Jak-STAT signaling pathway" (Fig. 1a). To make a connection between the enriched lncRNAs and mRNAs, we screened out the top 20 abundant mRNAs. As shown in Fig. 1b, majority of the first 20 abundant mRNAs were inflammatory responserelated genes, such as Chemokine ( $\mathrm{C}-\mathrm{X}-\mathrm{C}$ motif) ligand 8 (CXCL8), Ferritin light chain (FTL), S100A8, Chemokine (C-X-C motif) ligand 6 (CXCL6), Cathepsin $\mathrm{S}$ (CTSS), Galectin-3 (LGALS3), Elongation factor 1-alpha $1(E E F 1 A)$, thioredoxin $(\operatorname{tr} x A)$, Apolipoprotein $\mathrm{E}(A P O E)$, C-type lysozyme enzyme ( $L Y Z)$, Leukocyte surface antigen (CD53), Chemokine ligand $\mathrm{x}(C C L x)$, Superoxide dismutase 2 (SOD2), and Chemokine (C-X-C motif) ligand 2 (CXCL2). These data indicate that the PAMs are enriched in immune response-related lncRNAs and their predicted target genes, which allow them to respond quickly to invading organisms.

\section{Validation the susceptibility of PAMs to PRRSV infection}

To perform the functional analysis of porcine lncRNAs, we established in vitro model of inflammation by incubating PAMs with a high-pathogenic PRRSV strain HuN4. Treated cells were then collected for RNA sequence analysis. To confirm whether the PAMs were successfully infected by PRRSV, virus replication was determined by $\mathrm{TCID}_{50}$ and Western blot assay. The results showed that the virus titer obtained from PAMs 
a

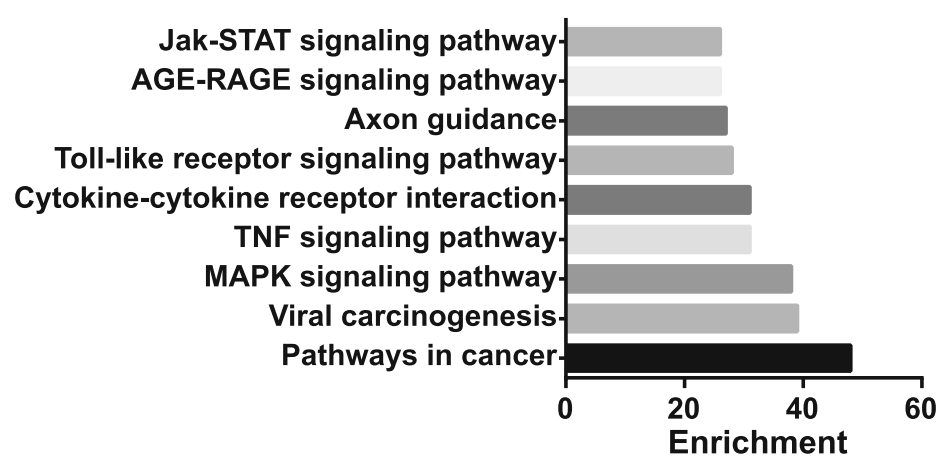

b

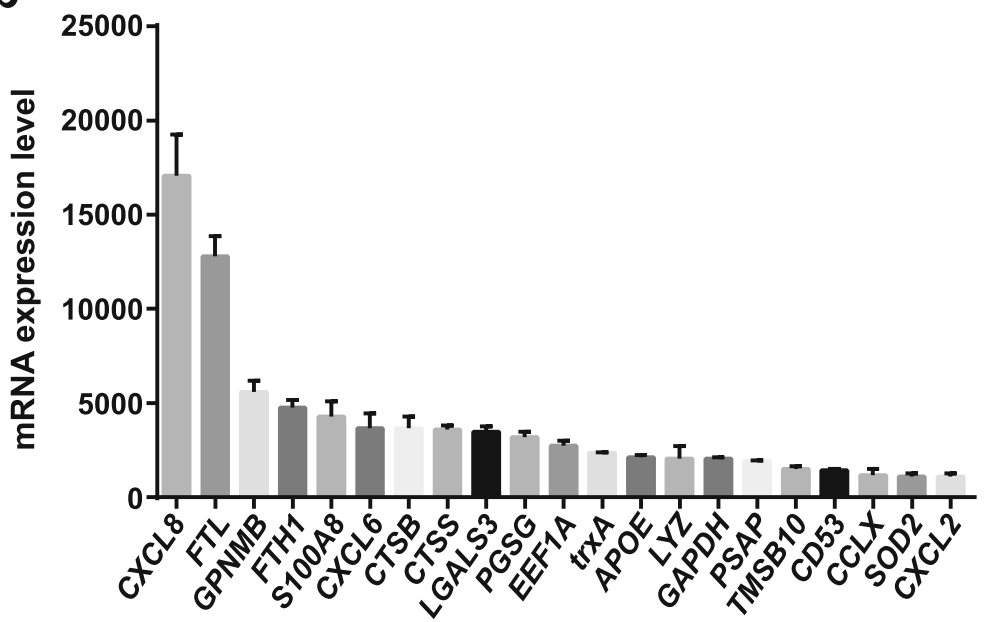

Fig. 1 The expression profiles of endogenous IncRNAs and mRNAs in PAMs. a KEGG pathway analysis of IncRNAs in PAMs. b Top 20 abundant mRNAs in PAMs

supernatant was $5.25 \log 10 \mathrm{TCID}_{50} / \mathrm{ml}$ (Fig. 2a) and the expression of $\mathrm{N}$ protein was also confirmed in PRRSVtreated PAMs (Fig. 2b and Additional file 1).

\section{LncRNA expression profile in PRRSV-infected PAMs}

Freshly isolated PAMs were treated with PRRSV HuN4 or mock, and followed by RNA-seq methodology. The results showed that the expression levels of 86 relatively abundant lncRNAs (FPKM > 1) were significantly altered upon virus incubation (Fig. 3a and Additional file 2).

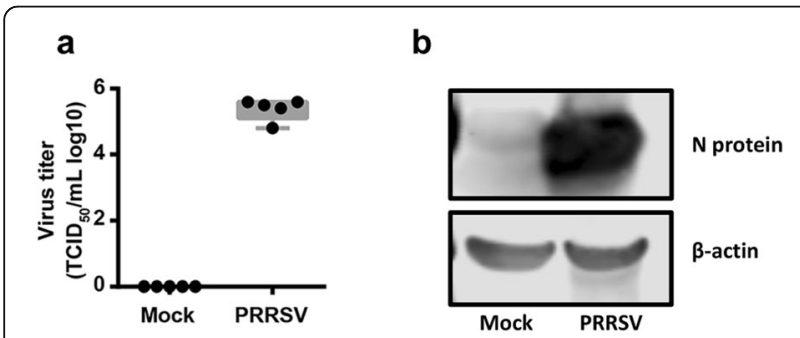

Fig. 2 Validation of PAMs susceptibility to PRRSV infection. a The virus titer determined by $\mathrm{TCID}_{50}$. $\mathbf{b}$ The expression of viral $\mathrm{N}$ protein determined by Western-blotting
Among them, 33 lncRNAs were upregulated and 53 were downregulated (fold change $>2.0, P$-value $<0.05$ ). To further employ the differentially expressed (DE) lncRNAs upon PRRSV infection, the unsupervised hierarchical clustering analysis was used. Heat maps showed overt self-segregated clusters in PAMs treated with PRRSV and mock (Fig. 3b). To further verify the accuracy of RNA-seq results, we tested four upregulated lncRNAs (XLOC-022131, XLOC-022175, XLOC019295, and XLOC-007149) and one downregulated lncRNA (XLOC-017089) by quantitative PCR (qPCR). As shown in Fig. 3c and Table 1, XLOC-022131, XLOC022175, XLOC-019295, and XLOC-007149 were significantly upregulated and XLOC-017089 was significantly downregulated, indicating the accuracy of RNA-seq data.

We next performed GO and KEGG pathway analyses to evaluate the function of DE IncRNAs. As shown in Fig. $3 d$, the upregulated lncRNAs were primarily associated with "NF-kB signaling pathway", "toll-like receptor signaling pathway", "MAPK signaling pathway", "RIG-Ilike receptor signaling pathway", "Jak-STAT signaling pathway", and "TNF signaling pathway". The 
a

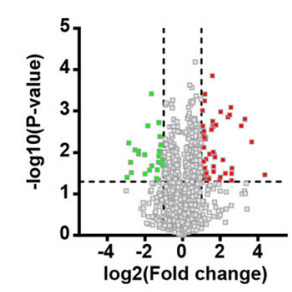

b

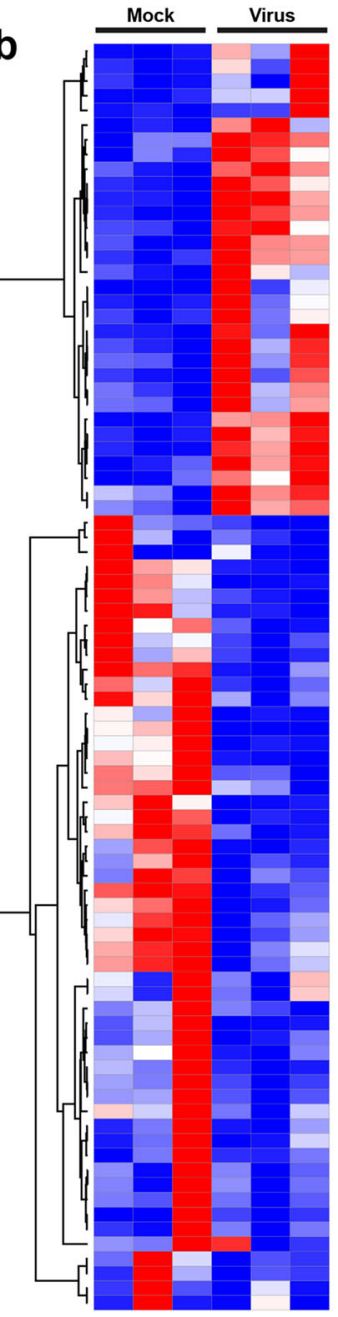

C

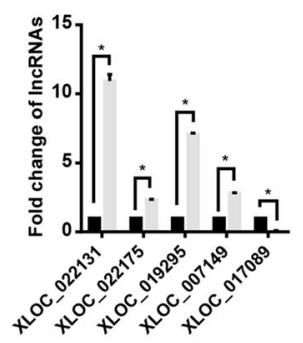

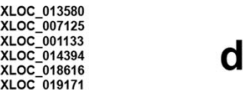
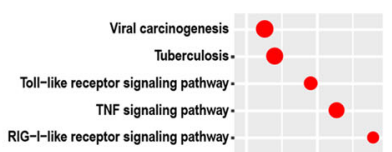

Row Max

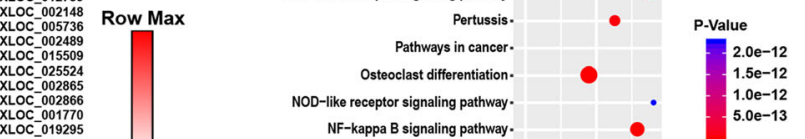

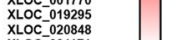

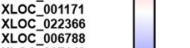

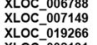

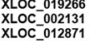

XLOC_012871

XLOC-11691

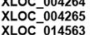

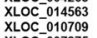

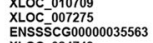

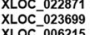

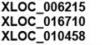

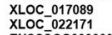

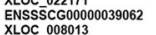

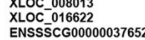

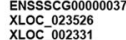

XLoC-002331
XLOC- 0153238

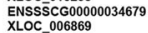

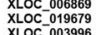

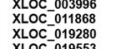

XLOC 019553
$\times$ LOC 017213
XLOC 011894

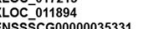

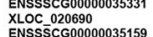

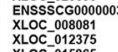

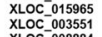

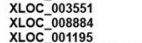

ENss5cc000000034497

ENSSSCGC0000003
XLCOCO08458
XLOC_00192

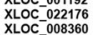

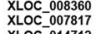

L L

XLoc-025072

ENSSSCGCO0000038539

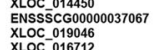

Fig. 3 DE IncRNAs in PAMs upon PRRSV infection. a Volcano plots of DE IncRNAs between mock and PRRSV-infected PAMs. Red or green points represent the DE IncRNAs with statistical significance $(P<0.05)$. $\mathbf{b}$ Unsupervised hierarchical clustering and heat map of IncRNA expression between mock and PRRSV-infected PAMs. Each column represents a sample and each row represents a IncRNA. Red color indicates relatively high expression and blue color indicates relatively low expression. c Verification of IncRNAs expression in mock and PRRSV-infected PAMs. Expression levels of four upregulated and one downregulated IncRNAs were verified by qPCR and normalized to $\beta$-actin. The results were presented as the fold change to the corresponding uninfected control $\left(^{*}, P<0.05\right)$. $\mathbf{d}$ and e Pathway analysis of DE IncRNAs after PRRSV infection. The dot plots represent the enrichment of the IncRNAs in each pathway. The color of each dot corresponds to the $P$-value. The size of each dot shows the number of IncRNAs. The horizontal axis represents the enrichment level of the pathways. A higher enrichment level means a larger change after PRRSV infection when the $P$-values are the same. Pathway enrichment of upregulated IncRNAs (d) and downregulated IncRNAs (e) was shown respectively. Results are representative of three independent experiments (means \pm SD). ${ }^{*}, P<0.05$. The $P$ value was calculated using Student's t-test 
Table 1 Primers used for qPCR

\begin{tabular}{ll}
\hline Primer & Sequence (5'-3') \\
\hline Porcine -actin-F & CTTCCTGGGCATGGAGTCC \\
Porcine -actin-R & GGCGCGATGATCTTGATCTTC \\
Porcine-IFI6-F & GAAGACGCTCTGAGGACAAC \\
Porcine-IFI6-R & CGGTTGTGAAGCCCAGAG \\
Porcine-CXCL2-F & GGAAGTTTGTCTCAACCCCGC \\
Porcine-CXCL2-R & AGCCAGTAAGTTTCCTCCATCTC \\
Porcine-CD163-F & ATGGGCTAATTCCAGTGCAG \\
Porcine-CD163-R & GATCCATCTGAGCAAGTCACTCCA \\
XLOC-017089-F & CTTAACCTACTGAGCCAAGCC \\
XLOC-017089-R & ATGTACTTTACCAGATTTGTCATGAAA \\
XLOC-022175-F & ACGAATAGTGAGTGTGAGGGC \\
XLOC-022175-R & GACAGAATGACTCTACTCACACG \\
XLOC-019295-F & GATCTCGTTGGCTTCTCATAG \\
XLOC-019295-R & GAGCTTCCTCTGTCATACTTGG \\
XLOC-007149-F & CCTTGCTTCTGTTCTCCTGG \\
XLOC-007149-R & GTCCTCATTCTCTTCCTCGG \\
XLOC-002131-F & GGTCTCCATGTCATTCCGATG \\
XLOC-002131-R & TTACTCACTTGCTCTGCCAC \\
\hline
\end{tabular}

downregulated lncRNAs were enriched in "PI3K-Akt signaling pathway", "chemokine signaling pathway", and "MAPK signaling pathway" (Fig. 3e). The GO and KEGG pathway patterns suggest that DE IncRNAs are largely associated with the inflammation- and pathogen infection-induced immune responses upon PRRSV infection, indicating that these DE InRNAs may play critical roles in regulating the virus-induced inflammatory responses in PAMs.

\section{Gene expression signature in PRRSV-infected PAMs}

Based on the analysis of RNA-seq data, we also generated volcano plots to visualize the DE profile of proteincoding mRNAs. Totally 406 mRNAs were differentially expressed (126 upregulated and 280 downregulated mRNAs) in PAMs after PRRSV treatment (Fig. 4a and Additional file 3). The hierarchical clustering analysis revealed that $\mathrm{DE}$ mRNAs were precisely distinguished by PRRSV-infected and mock-treated PAMs (Fig. 4b). Furthermore, we performed the GO and KEGG pathway analyses for DE mRNAs. Figure 4c showed that the upregulated genes were enriched in "TNF signaling pathway", "cytokine-cytokine receptor interaction", "chemokine signaling pathway", "rheumatoid arthritis", "Tolllike receptor signaling pathway", and "inflammatory bowel disease". The most downregulated genes were enriched in pathways including "metabolic pathways" and "complement and coagulation cascades" (Fig. 4d). The pathway patterns determined by GO and KEGG analyses indicate that these DE genes are dominantly related to pathogen-induced inflammatory immune responses. Combined with lncRNA profiles, these results implicate that the DE IncRNAs may have indispensable regulatory functions in the DE genes in PAMs upon virus stimulation.

\section{Identification of the regulatory function of IncRNAs in immune responses}

Correlation network is an innovative tool to better integrate the co-expressed genes with the regulatory functions of lncRNAs. Here the correlation analysis encompassed 86 lncRNA nodes and 406 mRNA nodes with significant changes in the virus-stimulated PAMs. By using a Pearson Correlation, we identified numerous sets of DE genes with temporal co-expression patterns (Additional file 4). To annotate the function of DE lncRNAs, we selected four immune-related genes including CXCL2, IFI6, IFITM1, and CD163 as modules to unearth the corresponding regulatory lncRNAs.

CXCL2, as a powerful chemoattractant, is exclusively secreted by monocytes and macrophages, involving in neutrophil recruitment and other immune responses during inflammation and wound healing [24]. For the CXCL2-lncRNAs network, 17 lncRNAs positively correlated with CXCL2 expression were upregulated, while 24 IncRNAs negatively correlated with CXCL2 expression were downregulated in PRRSV-infected cells (Fig. 5a). The CXCL2 module indicates that significantly enriched lncRNAs may play a dual role in the macrophages for microbial killing and initiating tissue repair [25].

Numerous IFNs stimulated genes, transcriptionally induced as the downstream signaling molecule of IFNs, can inhibit the infection of multiple viral families. Among these antiviral genes, the IFN-inducible protein (IFI) family and IFN-induced transmembrane protein (IFITM) family have broad-spectrum antiviral functions. The IFITM family consists of IFITM1, 2, 3, 5, and 6 [26], which can block the replication and infection of enveloped viruses [27]. IFI6 can target viral replication at the endoplasmic reticulum or distinct membranous organelles [28]. Here, the IFI6 and IFITM1 were selected to build the lncRNA/mRNA co-expression network. For the IFN-inducible genes network, 27 lncRNAs positively correlated with IFITM1/IFI6 expression were upregulated, while 13 lncRNAs negatively correlated with IFIT M1/IFI6 expression were downregulated in PRRSVinfected cells (Fig. 5b). Our results indicate that the DE lncRNAs associated with IFN-inducible genes have the potential to regulate the antiviral functions in macrophages.

CD163 has been identified as the essential receptor that mediates PRRSV entry $[29,30]$. Therefore, the CD163-lncRNAs correlation network was analyzed. As shown in Fig. 5c, 16 lncRNAs negatively correlated with 


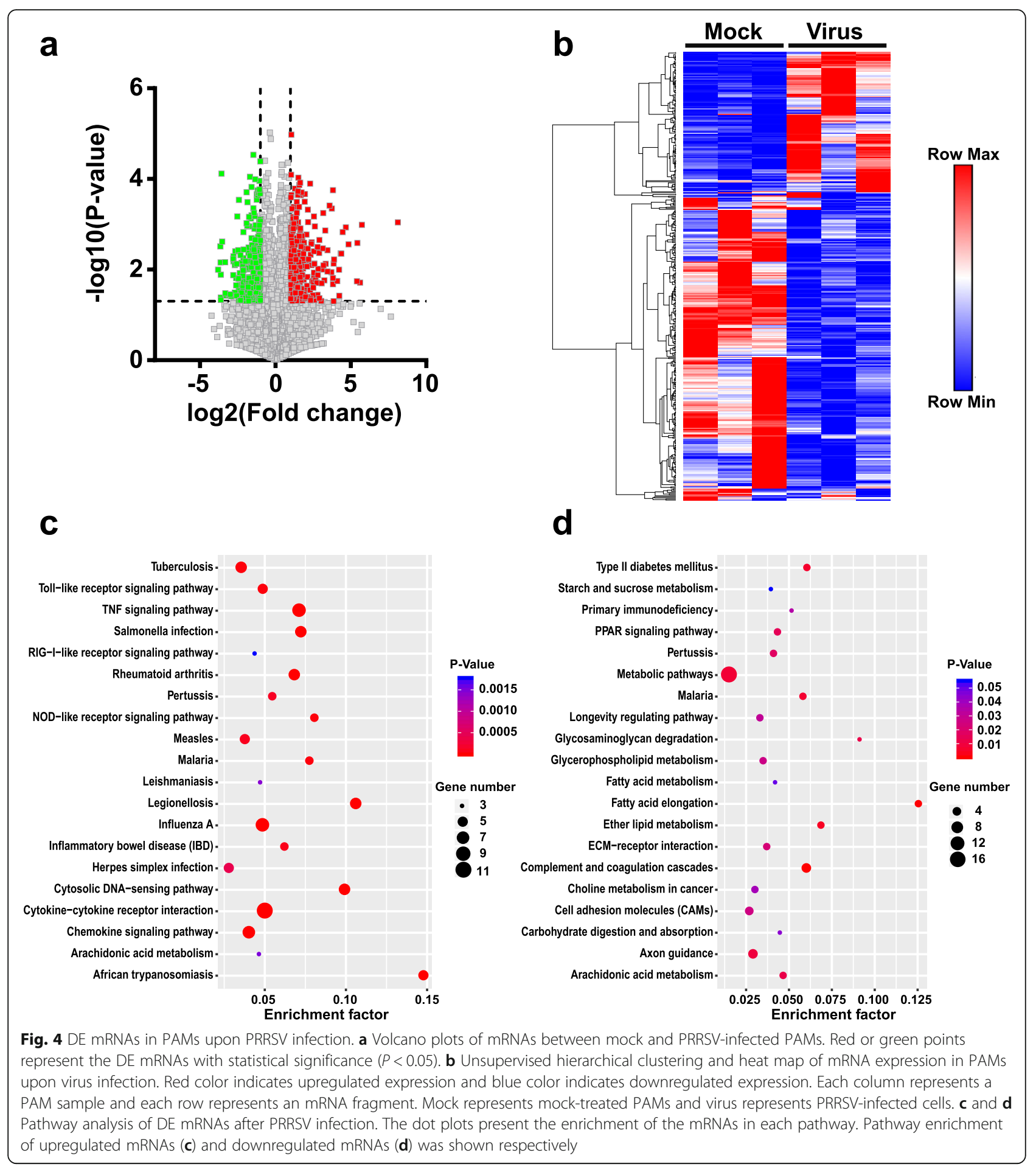

CD163 expression were upregulated, while 22 lncRNAs positively correlated with CD163 expression were downregulated in PRRSV infected cells. The CD163 module suggests that DE IncRNAs may play important roles in PRRSV entry by regulating the expression level of CD163.
Validation of differentially expressed IncRNAs by qPCR To verify our correlation networks, qPCR was used to examine three significantly differentially expressed lncRNA-mRNA pairs in PRRSV-infected PAMs. As shown in Fig. 6a and Table 1, all three lncRNA-mRNA pairs, including lncRNA XLOC-022175 vs CXCL2 (co- 


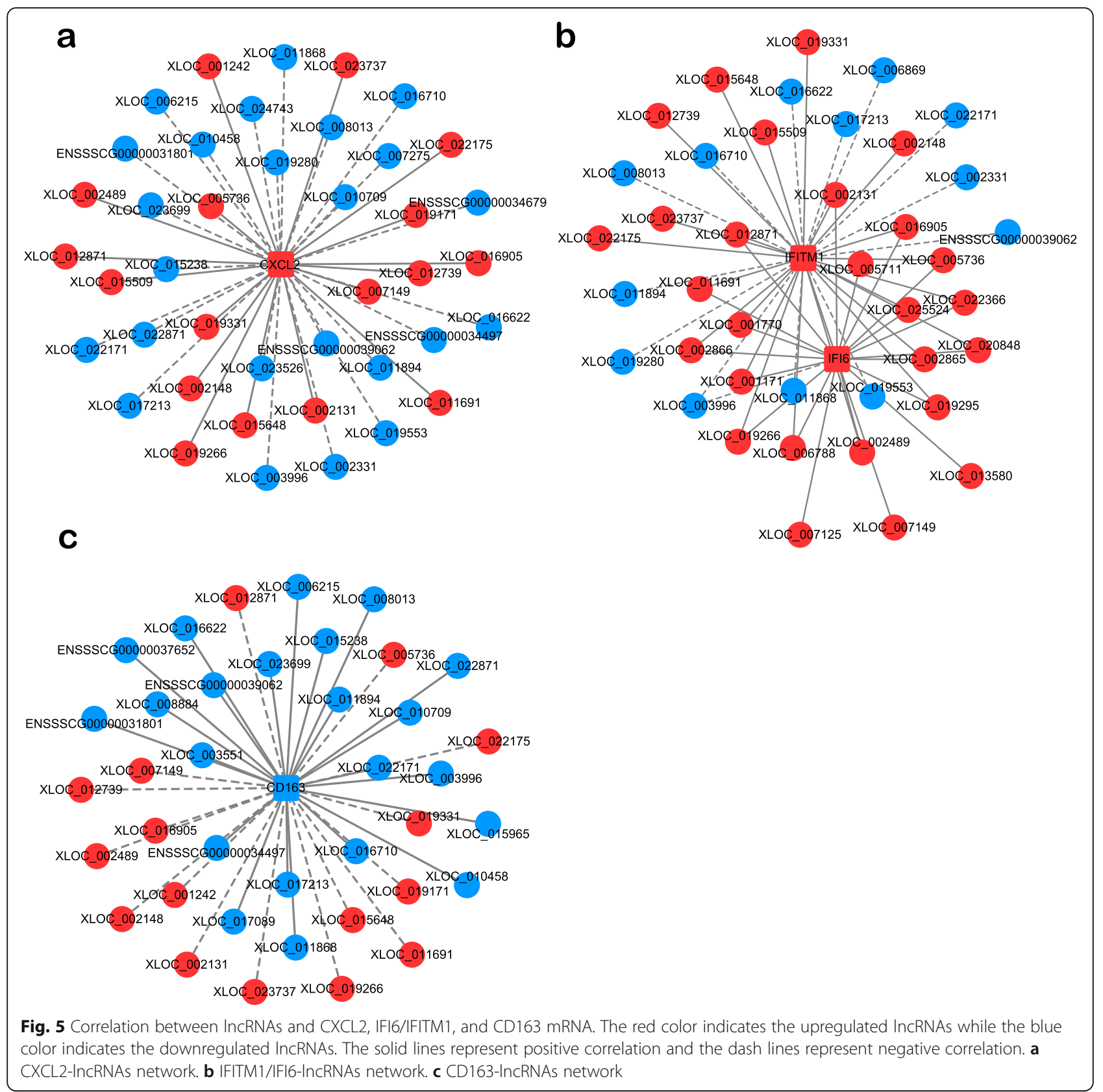

upregulated), XLOC-019295 vs IFI6 (co-upregulated), and XLOC-017089 vs CD163 (co-downregulated) exhibited significant changes after virus infection, which coincided with the predicted correlation networks. Additionally, the relationship between these three lncRNAs and their neighboring genes was analyzed based on the genomic location and transcriptome expression profile (Fig. $6 \mathrm{~b}$ and Table 2). Sequence analysis revealed that XLOC-22175 as a sense IncRNA located at the CXCL2 genomic locus, suggesting that the upregulated XLOC-022175 may promote the expression of
CXCL2 (Fig. 6a i). Sequence databases also showed that both XLOC-019295 and XLOC-017089 were antisense lncRNAs, and located near the genomic region of either IFI6 or CD163, indicating that both lncRNAs may facilitate the expression of IFI6 and CD163, respectively (Fig. $6 \mathrm{a}$ ii and iii). Interestingly, XLOC-019295 was predicted to be a trans-regulatory element for the expression of distant gene IFITM1 (Table 2). Although the correlations of these three IncRNA-mRNA pairs were confirmed by $\mathrm{qPCR}$, their potential immune regulatory function needs further investigation. 

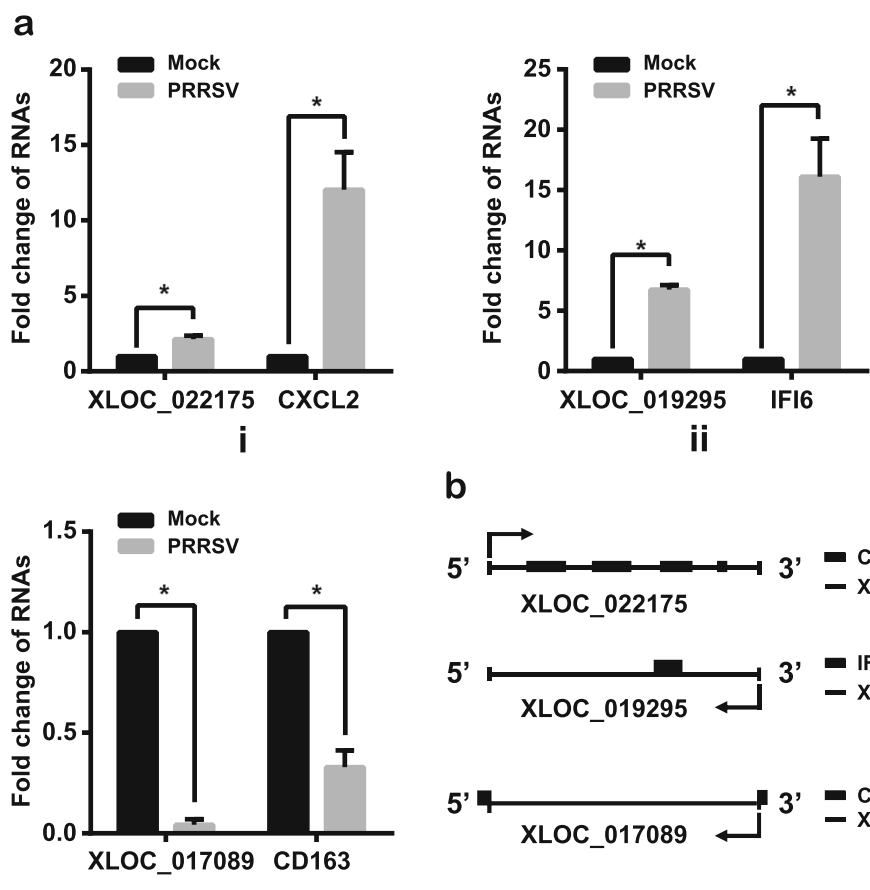

b
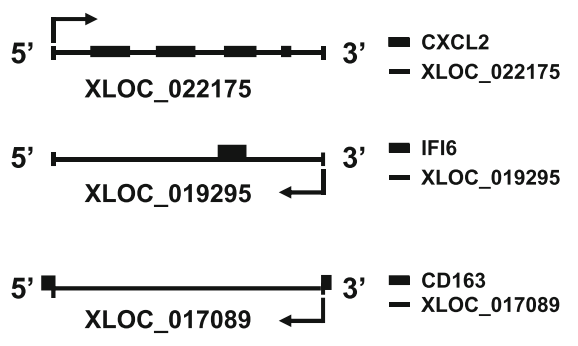

iii

Fig. 6 Verification of the correlation between IncRNAs and mRNAs. a PAMs were infected with PRRSV at a MOI of 5. At 24 hpi, total RNA was extracted and subjected to GPCR for detecting the expression levels of transcripts as indicated. (i) XLOC_022175 and CXCL2 mRNA. (ii) XLOC_019295 and IFI6 mRNA. (iii) XLOC_017089 and CD163 mRNA. b The location of IncRNAs and target mRNAs respectively. Results are representative of three independent experiments (means $\pm \mathrm{SD}$ ). ${ }^{*}, P<0.05$. The $P$ value was calculated using Student's $t$-test

\section{Discussion}

There are numerous lncRNAs available in public databases concerning their sequence, expression, and function [31-33]. The recent growth of next-generation sequencing technology has accelerated the research progress of lncRNAs. Although tens of thousands of IncRNAs have been discovered, only a small fraction of lncRNAs have been found to regulate gene expression in mouse and human. Even more noteworthy is the fact that only $15 \%$ of mouse lncRNAs are expressed in human and vice versa, suggesting dramatic speciesspecificity [34]. However, there are few databases about porcine lncRNAs and their potential functions [35-38], resulting in majority of porcine lncRNAs remains to be unidentified. Macrophages, as one of the critical regulators of both normal homeostasis and pathology, have attracted much attention from researchers to investigate the molecular mechanisms responsible for their function. In this study, the high-throughput RNA-seq method was utilized to investigate the detailed information about lncRNAs in porcine macrophages. We found 2142 lncRNAs abundantly expressed in PAMs. To describe the biological functions of these lncRNAs, GO and KEGG pathways are commonly introduced [39, 40]. GO and KEGG pathway analyses revealed that the regulatory signaling pathways of these lncRNAs mainly enriched in IFNs and cytokines production, indicating that lncRNAs may have a tight connection with immune response in porcine macrophages.

To further determine the regulatory function of these lncRNAs, we incubated PAMs with PRRSV to induce the inflammation model [41]. According to RNA-seq data, we defined a set of 86 DE lncRNAs in PAMs after PRRSV infection. GO and KEGG pathway enrichment analyses revealed that most DE lncRNAs and proteincoding genes were associated with inflammatory

Table $\mathbf{2}$ LncRNA-mRNA pairs with Pearson Correlation coefficients $>0.9$

\begin{tabular}{lllll}
\hline LncRNA & Correlation coefficients. & Target gene & KEGG name & Function \\
\hline XLOC_022175 & 0.949422683 & ENSSSCG00000008959 & CXCL2 & cis \\
XLOC_019295 & 0.997191011 & ENSSSCG00000034570 & IFI6 & cis \\
& 0.941706488 & ENSSSCG00000014565 & IFITM1 & trans \\
XLOC_017089 & 0.952479684 & ENSSSCG00000033146 & CD163 & cis \\
\hline
\end{tabular}


pathways in PAMs. Since the network analysis can provide a global view of all possible lncRNAs-mRNA expression associations based on the different immune background, here, the network analysis was used to predict the functional annotations of DE lncRNAs. We observed the cooperative expression relationships between four protein-coding genes (CXCL2, IFI6, IFITM1, and CD163) and their predicted regulator lncRNAs. Likewise, other studies have also employed a similar strategy to identify functional lncRNA signatures in different types of cancer [42, 43].

LncRNAs are recognized as transcriptional regulators that may be involved in cis or trans regulation of genes located in their vicinity or at distant loci [44]. In cis, the sense and antisense lncRNAs interact with genes transcribed from the same DNA region; whereas in trans, the interaction is with genes located at distant loci or even at other chromosomes $[45,46]$. Here, after validation by qPCR, three selected lncRNAs were showed to have a positively correlated expression with their predicted target genes in PAMs. By using sequence alignment between full-length lncRNAs and nearby mRNAs, we especially considered protein coding gene loci which act as hosts for IncRNA transcripts. In other published literature about lncRNA profiles upon PRRSV infection, there is no such detailed information provided [37, 38, $47,48]$. Only Zeng et al. revealed that DE lncRNA XR 297549.1 induced by PRRSV infection was predicted to both cis-regulate and trans-regulate its neighboring gene, prostaglandin-endoperoxide synthase 2 [49]. In this study, XLOC-022175, a sense lncRNA in cisregulatory fashion, was predicted to be positively correlated with CXCL2, which is mainly released from activated macrophages and capable of attracting CXCR2 + cells, such as neutrophils to sites of inflammation [50]. Our data also showed that IFI6 and IFITM1 were predicted to be the cis- and trans-regulated target genes of XLOC-019295, respectively. Both IFI6 and IFIT M1 belong to IFN-stimulated genes with antiviral activity [26]. IFITM1, a virus-restriction factor, have been proved to restrict the replication of several viruses, including PRRSV, PRV, PCV2, and SIV [51-53]. XLOC017089 was identified as an antisense lncRNA and predicted to act in cis to regulate CD163 expression, which has been determined to be the essential receptor for PRRSV $[16,54]$. These presented data suggest that our proposed method can better reveal the function of lncRNAs not only through the correlation network of different expression but also through cis or trans regulatory mode of lncRNAs located. By using the similar methodology, other cutting-edge studies have indicated that lncRNAs regulate the function of their target genes through cis- and trans-acting, such as Xist, PTEN, and HOTAIR [43, 55, 56].
Taken together, our findings provide novel insights on functional characterization of lncRNAs in innate immune responses of porcine macrophages. However, the detail mechanisms of these core lncRNAs need to be further studied and established. We need precisely characterize these lncRNAs phenotypes and identify its functions during in vitro and in vivo infection. Technically we need conduct genetic modification systems to answer whether lncRNAs XLOC-022175, XLOC-019295, and XLOC-017089 play critical roles in inflammation, antiviral response, and PRRSV infection. More specifically, we will investigate whether IncRNA XLOC-022175 can regulate CXCL2 secretion to recruit neutrophils to sites of PRRSV infection, whether XLOC-019295 plays a duel role in host defense against infectious diseases, and whether XLOC-017089 can modify PRRSV infection through regulating the expression of the scavenger receptor CD163.

\section{Conclusion}

In summary, RNA-seq can be a useful tool to analyze the expression profiles of lncRNAs and their regulatory function. Here, the transcriptome analysis revealed that a total of $86 \mathrm{DE}$ lncRNAs and $406 \mathrm{DE}$ protein-coding mRNAs were mainly involved in PRRSV-induced inflammation and immune responses in PAMs. Correlated expression network analysis identified that numerous lncRNAs participated in the innate immune responses. Correlation analysis of DE lncRNAs revealed that the predicted target genes of XLOC-022175, XLOC-019295, and XLOC-017089 were related to the inflammation and antiviral signaling pathways. These DE lncRNAs comprised a valuable atlas that could be used to connect lncRNAs expression with viral pathogenesis. Further studies are required to investigate the regulatory roles of these lncRNAs during virus infection.

\section{Methods}

A full step-by-step process can be found in Additional file 5.

\section{Animals, cells and viruses}

Three five-week-old SPF Landrace pigs were from Harbin Veterinary Research Institute, Chinese Academy of Agricultural Sciences. Pigs were euthanized by Pentobarbital sodium intravenously $(100 \mathrm{mg} / \mathrm{kg})$ and sacrificed. Immediately, lungs were collected sterilely and primary PAMs were freshly isolated. Animal experiments were approved by the Animal Care and Use Committee of Harbin Veterinary Research Institute of Chinese Academy of Agricultural Sciences (Approval ID: 200720-01) and experiments were performed according to the regulations and guidelines established by this committee. PAMs were cultured in DMEM (Life 
Technologies, USA) supplemented with $10 \%$ heatinactivated FBS (Hyclone), $100 \mathrm{U} / \mathrm{ml}$ penicillin, $100 \mu \mathrm{g} /$ $\mathrm{mL}$ streptomycin at $37^{\circ} \mathrm{C}$ in a $5 \% \mathrm{CO}_{2}$ incubator (Thermo Scientific, USA). Marc-145 cells, a monkey kidney cell line, were maintained in DMEM as well. The PRRSV North American-like strain HuN4 (GenBank accession number EF635006), a high pathogenic PRRSV strain [57] was cultured in Marc-145 cells and PAMs as indicated.

\section{$\mathrm{TCID}_{50}$ assay}

Median tissue culture infectious does (TCID50) assay was performed on Marc-145 cells as previously described [57]. Virus samples were collected by centrifugation for $10 \mathrm{~min}$ at $8000 \times \mathrm{g}$ and diluted by 10 -fold serial dilution. Cell monolayers were inoculated with diluted viruses and cultured for 5-6 days until the appearance of cytopathic effect. The virus titers were calculated by Reed \& Muench method.

\section{Western blotting}

Western blotting analysis was performed as described previously with a slight modification [58]. PAMs were inoculated with PRRSV or mock at a MOI of 5 . At $24 \mathrm{~h}$ postinfection, cells were lysed with Pierce IP lysis buffer (Thermo Scientific, Rockford, IL). The cell lysates were separated by SDS-PAGE under reducing condition and transferred onto PVDF membrane. After blocking, the membrane was incubated with the appropriate primary and secondary antibodies. The membranes were scanned and analyzed using an Odyssey instrument (Li-Cor Biosciences). The blotting mAb against PRRSV nucleocapsid $(\mathrm{N})$ protein was prepared in our laboratory. Antiactin mAb (C4) was purchased from Santa Cruz Biotechnology (Santa Cruz, CA). The IRDye-conjugate secondary antibody was from Li-Cor Biosciences (Lincoln, NE).

\section{Total RNA extraction and quantitative PCR}

Total RNA was extracted from cells with TRIzol (Invitrogen, SIGMA), and was then reverse transcribed into cDNA using PrimeScript RT reagent Kit with gDNA Eraser by random primers (TaKaRa, Japan) according to the manufacturer's instructions. Quantitative PCR (qPCR) was carried out in a QuantStudio 5 system (Applied Biosystems) using SYBR premix Ex Taq (TaKaRa, Japan). Fold changes were determined using the cycle threshold $(\Delta \Delta \mathrm{CT})$ method [59].

Whole transcriptome library preparation and sequencing RNA transcripts were purified from total RNA with oligo (dT)-attached magnetic beads and fragmented into short fragments. First-strand cDNA and second-strand cDNA were subsequently synthesized using random hexamer primer. The cDNA fragments were purified and resolved with EB buffer for terminal repair, the addition of single nucleotide A and adapters. After size selecting and retrieving by AMPure XP beads, the products were used for PCR amplification to obtain the library. Agilent 2100 Bioanalyzer and ABI StepOnePlus Real-Time PCR System were used to assess the qualification and quantification of those libraries. The eligible libraries were sequenced using Illumina HiSeqTM X TEN.

\section{RNA-seq data analysis}

Clean reads were obtained by removing reads with adaptors, reads with unknown bases ( $\mathrm{N}$ bases more than $5 \%$ ), and low quality reads (bases qualities lower than 10). Next, HISAT [60] tools were used to map clean reads to the indexed reference transcriptome. Gene expression levels were normalized using FPKM method by RSEM $[61,62]$. Differentially expressed genes were screened by Edge $R$ [63] with the criteria of fold change $\geq 2$ and $\mathrm{FDR} \leq 0.01$.

\section{Function prediction of IncRNAs}

To determine the expression profile of lncRNAs, RNA transcripts were reconstructed and identified by mapped reads using StringTie [64] and cuffcompare [65]. The known IncRNAs were acquired by comparing the assembled transcripts with annotated IncRNAs from NCBI, Ensembl, and UCSC. To predict new IncRNAs, we only retained transcripts longer than 200 nucleotides, more than one exon, and optimum expression threshold of FPKM $>0.5$ in at least one sample. The software CNCI [66], CPC [67], and txCdsPredict were used to predict the protein-coding potential of new transcripts with default parameters. Only transcripts that did not pass the protein-coding score test $(\mathrm{CPC}$ score $<0$, CNCI score $<0$, txCdsPredict score $<500)$ were predicted as new lncRNAs. The expression level of lncRNA was calculated using FPKM within the software RSEM. Transcripts with an FDR $<0.01$ and fold change $\geq 2$ were identified as significantly DE lncRNAs using EdgeR. The potential target genes of DE lncRNAs in cis- and transregulatory effects were predicted. The neighboring protein-coding genes were predicted as the cis target genes. To predict trans target genes, according to the FPKM values of the different expression of lncRNAs and mRNAs in all the samples, the Pearson Correlation coefficient between the lncRNAs and the mRNAs were calculated, and the threshold for positive correlation was set to Pearson Correlation $>0.8$.

\section{Differential expression analysis of IncRNAs and mRNAs}

For each sample, FPKM was a normalized estimation of both mRNA and IncRNA based on RNA-seq data. 
FPKM was calculated from the number of reads that mapped to each particular gene sequence taking into account the gene length and the sequencing depth. By using DESeq [68] package in $\mathrm{R}$ language and $t$-test, mRNA and lncRNA differential expression analyses were performed for all pairwise comparisons between PRRSVinfected groups and mock-treated groups. A corrected $P$-value $<0.05$ by Student's $t$-test with BenjaminiHochberg FDR adjustment was used as the cut-off for significantly DE genes.

\section{Heatmap and functional annotation of differentially expressed transcripts}

To show fold changes of the DE transcripts between groups, hierarchical heatmaps were clustered by the one minus Pearson Correlation method and generated by MORPHEUS (https://software.broadinstitute.org/ morpheus). To gain insight into the functions of DE transcripts, the associated GO terms were identified using DAVID Bioinformatics Resources (version 6.7, http://david.abcc.ncifcrf.gov/). Specifically, GeneRatio and BgRation were used to classify the GO category. DAVID software was used to analyze the signaling pathways of DE genes according to KEGG. Two-sided Fisher's exact test and $\mathrm{x}^{2}$ test were used to select significant pathways, and the FDR was calculated to correct the $P$-value.

\section{LncRNA-mRNA correlation network}

The lncRNA-mRNA correlation network was constructed by correlation calculation based on the values of the normalized signal intensity of specific expression in the DE genes. For each pair of lncRNA-mRNA, the Pearson Correlation was calculated to determine the significance of correlation and the correlation value cut-off was 0.8. Correlation degrees of lncRNAs and mRNAs were calculated by counting their correlated counterparts. The $P$-value denoted the significant level of gene co-expression and the threshold of significance was $P$ value $<0.05$.

\section{Statistical analysis}

For next-generation sequencing data, all the experimental condition was independently repeated three times and in each of three biological repetitions, three technical replicas were made. Clean reads were analyzed by Edge $\mathrm{R}$ and the criteria of fold change $\geq 2$ and FDR $\leq$ 0.01 . Correlation network data were calculated by Pearson Correlation coefficient and the correlation value cut-off was 0.8 and the threshold of significance was $P$ value $<0.05$. All statistical data were expressed as mean \pm standard deviation (SD) of three independent experiments and analyzed using Student's $t$-test. A $P$-value of $<0.05$ was considered statistically significant.

\section{Abbreviations}

APOE: Apolipoprotein E; CCL: Chemokine ligand; CD53: Leukocyte surface antigen; CD163: Scavenger receptor; circRNAs: Circular RNAs; CTSS: Cathepsin S; CXCL: Chemokine (C-X-C motif) ligand; CXCR: Chemokine (C-X-C motif) receptor; DE: Differently expressed; EEF1A: Elongation factor 1-alpha 1; FDR: False discovery rate; FPKM: Fragments Per Kiolbase MillionTPM;

FTL: Ferritin light chain; GO: Gene ontology; hnRNP: Heterogeneous nuclear ribonucleoprotein; hpi: Hours post-incubation; IFI: IFN-inducible protein; IFIT M: IFN-induced transmembrane protein; IFN: Interferon; IL: Interleukin; IRF: Interferon regulatory factor; ISG: Interferon-stimulated gene; KEGG: Kyoto Encyclopedia of Genes and Genomes; LGALS3: Galectin-3; LncRNA: Long noncoding RNA; LYZ: C-type lysozyme enzyme; miRNA: Micro RNA; MOI: Multiplicity of infection; OAS2: 2'-5' oligoadenylate synthase; PAM: Porcine alveolar macrophage; PRRSV: Porcine reproductive and respiratory syndrome virus; SD: Standard deviation; SOD2: Superoxide dismutase 2; SPF: Specific Pathogen Free; $\mathrm{TCID}_{50}$ : $50 \%$ tissue culture infective dose; TNFa: Tumor necrosis factor; trxA: Thioredoxin

\section{Supplementary Information}

The online version contains supplementary material available at https://doi. org/10.1186/s12864-021-07482-9.

\section{Additional file 1.}

Additional file 2.

Additional file 3.

Additional file 4.

Additional file 5.

\section{Acknowledgements}

Not applicable.

\section{Authors' contributions}

Y.W. and J.G. designed the experiments; J.G. carried out the experiments; J.G., Y.P., Y.X., W.Z., L.Z. and Y.W. analyzed the data; X.L., Z.T. and H.C. contributed materials/analysis tools and analyzed the data; Y.W. and J.G. wrote and reviewed the manuscript. All authors have read and approved the manuscript.

\section{Funding}

The research was supported by grants from the National Natural Science Foundation of China (31572497) and (31372416), and Heilongjiang Science Foundation for Distinguished Young Scholar (JC2016005). These funder(s) played no role in the design of the study and collection, analysis, and interpretation of data and in writing the manuscript.

\section{Availability of data and materials}

The RNA-seq data supporting the conclusions has been deposited into the NCBI Sequence Read Archive (SRA), under the accession number SRP278343 (https://www.ncbi.nlm.nih.gov/sra/?term=SRP278343).

\section{Declarations}

\section{Ethics approval and consent to participate}

The SPF Landrace pigs were owned by Harbin Veterinary Research Institute, Chinese Academy of Agricultural Sciences, Harbin, Heilongjiang Province, China. The entire procedure was carried out in strict accordance with the protocol approved by the Animal Care and Use Committee of Harbin Veterinary Research Institute of Chinese Academy of Agricultural Sciences (Approval ID: 200720-01) and experiments were performed according to the regulations and guidelines established by this committee.

Consent for publication

Not applicable.

Competing interests

The authors declare that they have no competing interests. 


\section{Received: 11 September 2020 Accepted: 25 February 2021} Published online: 12 March 2021

\section{References}

1. Venter JC, Adams MD, Myers EW, Li PW, Mural RJ, Sutton GG, Smith HO, Yandell M, Evans CA, Holt RA, et al. The sequence of the human genome. Science. 2001;291(5507):1304-51.

2. Cheetham SW, Gruhl F, Mattick JS, Dinger ME. Long noncoding RNAs and the genetics of cancer. Br J Cancer. 2013;108(12):2419-25.

3. Childs KS, Goodbourn S. Identification of novel co-repressor molecules for interferon regulatory Factor-2. Nucleic Acids Res. 2003:31(12):3016-26.

4. Werner MS, Sullivan MA, Shah RN, Nadadur RD, Grzybowski AT, Galat V, Moskowitz IP, Ruthenburg AJ. Chromatin-enriched IncRNAs can act as celltype specific activators of proximal gene transcription. Nat Struct Mol Biol. 2017:24(7):596-603.

5. Akerman I, Tu Z, Beucher A, Rolando DMY, Sauty-Colace C, Benazra M, Nakic $\mathrm{N}$, Yang J, Wang H, Pasquali L, et al. Human pancreatic beta cell IncRNAs control cell-specific regulatory networks. Cell Metab. 2017;25(2):400-11.

6. Cabili MN, Trapnell C, Goff L, Koziol M, Tazon-Vega B, Regev A, Rinn JL. Integrative annotation of human large intergenic noncoding RNAs reveals global properties and specific subclasses. Genes Dev. 2011;25(18):1915-27.

7. Crawford GE, Holt IE, Whittle J, Webb BD, Tai D, Davis S, Margulies EH, Chen Y, Bernat JA, Ginsburg D, et al. Genome-wide mapping of DNase hypersensitive sites using massively parallel signature sequencing (MPSS). Genome Res. 2006;16(1):123-31.

8. Jin F, Li Y, Dixon JR, Selvaraj S, Ye Z, Lee AY, Yen CA, Schmitt AD, Espinoza CA, Ren B. A high-resolution map of the three-dimensional chromatin interactome in human cells. Nature. 2013:503(7475):290-4.

9. Kretz M, Siprashvili Z, Chu C, Webster DE, Zehnder A, Qu K, Lee CS, Flockhart RJ, Groff AF, Chow J, et al. Control of somatic tissue differentiation by the long non-coding RNA TINCR. Nature. 2013;493(7431):231-5.

10. Tseng $Y Y$, Moriarity BS, Gong W, Akiyama R, Tiwari A, Kawakami H, Ronning P, Reuland B, Guenther K, Beadnell TC, et al. PVT1 dependence in cancer with MYC copy-number increase. Nature. 2014;512(7512):82-6.

11. Chen JF, Wu P, Xia R, Yang J, Huo XY, Gu DY, Tang CJ, De W, Yang F. STAT3-induced InCRNA HAGLROS overexpression contributes to the malignant progression of gastric cancer cells via mTOR signal-mediated inhibition of autophagy. Mol Cancer. 2018;17(1):6.

12. Liu SJ, Nowakowski TJ, Pollen AA, Lui JH, Horlbeck MA, Attenello FJ, He D, Weissman JS, Kriegstein AR, Diaz AA, et al. Single-cell analysis of long noncoding RNAs in the developing human neocortex. Genome Biol. 2016;17:67.

13. Carpenter S. Determining the function of long noncoding RNA in innate immunity. Methods Mol Biol. 2016;1390:183-95.

14. Li Z, Chao TC, Chang KY, Lin N, Patil VS, Shimizu C, Head SR, Burns JC, Rana TM. The long noncoding RNA THRIL regulates TNFalpha expression through its interaction with hnRNPL. Proc Natl Acad Sci U S A. 2014;111(3):1002-7.

15. Wensvoort G, Terpstra C, Pol JM, ter Laak EA, Bloemraad M, de Kluyver EP, Kragten C, van Buiten L, den Besten A, Wagenaar F, et al. Mystery swine disease in the Netherlands: the isolation of Lelystad virus. Vet Q. 1991;13(3): 121-30.

16. Lunney JK, Fang Y, Ladinig A, Chen NH, Li YH, Rowland B, Renukaradhya GJ. Porcine reproductive and respiratory syndrome virus (PRRSV): pathogenesis and interaction with the immune system. Annu Rev Anim Biosci. 2016:4: 129-54.

17. Nan $Y$, Wang R, Shen M, Faaberg KS, Samal SK, Zhang YJ. Induction of type interferons by a novel porcine reproductive and respiratory syndrome virus isolate. Virology. 2012;432(2):261-70

18. Brockmeier SL, Loving CL, Eberle KC, Hau SJ, Buckley A, Van Geelen A, Montiel NA, Nicholson T, Lager KM. Interferon alpha inhibits replication of a live-attenuated porcine reproductive and respiratory syndrome virus vaccine preventing development of an adaptive immune response in swine. Vet Microbiol. 2017;212:48-51.

19. An TQ, Li JN, Su CM, Yoo D. Molecular and cellular mechanisms for PRRSV pathogenesis and host response to infection. Virus Res. 2020;286:197980.

20. Gomez-Laguna J, Salguero FJ, Barranco I, Pallares FJ, Rodriguez-Gomez IM, Bernabe A, Carrasco L. Cytokine expression by macrophages in the lung of pigs infected with the porcine reproductive and respiratory syndrome virus. J Comp Pathol. 2010;142(1):51-60.

21. Alex Pasternak J, MacPhee DJ, Harding JCS. Fetal cytokine response to porcine reproductive and respiratory syndrome virus-2 infection. Cytokine. 2020;126:154883.
22. Qiao S, Feng L, Bao D, Guo J, Wan B, Xiao Z, Yang S, Zhang G. Porcine reproductive and respiratory syndrome virus and bacterial endotoxin act in synergy to amplify the inflammatory response of infected macrophages. Vet Microbiol. 2011;149(1-2):213-20.

23. Li R, Guo L, Gu W, Luo X, Zhang J, Xu Y, Tian Z, Feng L, Wang Y. Production of porcine TNFa by ADAM17-mediated cleavage negatively regulates porcine reproductive and respiratory syndrome virus infection. Immunol Res. 2016;64(3):711-20.

24. De Filippo K, Dudeck A, Hasenberg M, Nye E, van Rooijen N, Hartmann K, Gunzer M, Roers A, Hogg N. Mast cell and macrophage chemokines CXCL1/ CXCL2 control the early stage of neutrophil recruitment during tissue inflammation. Blood. 2013;121(24):4930-7.

25. Kobayashi Y. The role of chemokines in neutrophil biology. Front Biosci. 2008;13:2400-7.

26. Diamond MS, Farzan M. The broad-spectrum antiviral functions of IFIT and IFITM proteins. Nat Rev Immunol. 2013;13(1):46-57.

27. Han JH, Lee S, Park YS, Park JS, Kim KY, Lim JS, Oh KS, Yang Y. IFITM6 expression is increased in macrophages of tumor-bearing mice. Oncol Rep. 2011:25(2):531-6.

28. Richardson RB, Ohlson MB, Eitson JL, Kumar A, McDougal MB, Boys IN, Mar KB, De La Cruz-Rivera PC, Douglas C, Konopka G, et al. A CRISPR screen identifies IFI6 as an ER-resident interferon effector that blocks flavivirus replication. Nat Microbiol. 2018;3(11):1214-23.

29. Calvert JG, Slade DE, Shields SL, Jolie R, Mannan RM, Ankenbauer RG, Welch SK. CD163 expression confers susceptibility to porcine reproductive and respiratory syndrome viruses. J Virol. 2007;81(14):7371-9.

30. Prather RS, Whitworth KM, Schommer SK, Wells KD. Genetic engineering alveolar macrophages for host resistance to PRRSV. Vet Microbiol. 2017;209:124-9.

31. Li JH, Liu S, Zhou H, Qu LH, Yang JH. starBase v2.0: decoding miRNA-ceRNA, miRNA-ncRNA and protein-RNA interaction networks from large-scale CLIPSeq data. Nucleic Acids Res. 2014;42(Database issue):D92-7.

32. Bu D, Yu K, Sun $S$, Xie C, Skogerbo G, Miao R, Xiao H, Liao Q, Luo H, Zhao G, et al. NONCODE v3.0: integrative annotation of long noncoding RNAs. Nucleic Acids Res. 2012:40(Database issue):D210-5.

33. Volders PJ, Helsens K, Wang X, Menten B, Martens L, Gevaert K, Vandesompele J, Mestdagh P. LNCipedia: a database for annotated human IncRNA transcript sequences and structures. Nucleic Acids Res. 2013; 41(Database issue):D246-51.

34. Paralkar VR, Mishra T, Luan J, Yao Y, Kossenkov AV, Anderson SM, Dunagin M, Pimkin M, Gore M, Sun D, et al. Lineage and species-specific long noncoding RNAs during erythro-megakaryocytic development. Blood. 2014; 123(12):1927-37.

35. Zhao P, Zheng $X$, Feng $W$, Wang $H$, Kang H, Ning C, Du H, Yu Y, Li B, Zhao $Y$, et al. Profiling long noncoding RNA of multi-tissue transcriptome enhances porcine noncoding genome annotation. Epigenomics. 2018;10(3): 301-20.

36. Liang G, Yang Y, Li H, Yu H, Li X, Tang Z, Li K. LncRNAnet: a comprehensive Sus scrofa IncRNA database. Anim Genet. 2018;49(6):632-5.

37. Wu J, Peng X, Qiao M, Zhao H, Li M, Liu G, Mei S. Genome-wide analysis of long noncoding RNA and mRNA profiles in PRRSV-infected porcine alveolar macrophages. Genomics. 2020:112(2):1879-88.

38. Zhang J, Sun P, Gan L, Bai W, Wang Z, Li D, Cao Y, Fu Y, Li P, Bai X, et al. Genome-wide analysis of long noncoding RNA profiling in PRRSV-infected PAM cells by RNA sequencing. Sci Rep. 2017;7(1):4952.

39. Gene Ontology C. Gene Ontology consortium: going forward. Nucleic Acids Res. 2015;43(Database issue):D1049-56

40. Kanehisa M, Sato Y, Kawashima M, Furumichi M, Tanabe M. KEGG as a reference resource for gene and protein annotation. Nucleic Acids Res. 2016;44(D1):D457-62.

41. Crisci E, Fraile L, Montoya M. Cellular Innate Immunity against PRRSV and Swine Influenza Viruses. Vet Sci. 2019;6(1):26

42. Wang $P$, Ning S, Zhang Y, Li R, Ye J, Zhao Z, Zhi H, Wang T, Guo Z, Li X. Identification of IncRNA-associated competing triplets reveals global patterns and prognostic markers for cancer. Nucleic Acids Res. 2015;43(7): 3478-89.

43. Gupta RA, Shah N, Wang KC, Kim J, Horlings HM, Wong DJ, Tsai MC, Hung T, Argani P, Rinn JL, et al. Long non-coding RNA HOTAIR reprograms chromatin state to promote cancer metastasis. Nature. 2010;464(7291):1071-6.

44. Chen H, Du G, Song X, Li L. Non-coding transcripts from enhancers: new insights into enhancer activity and Gene expression regulation. Genomics Proteomics Bioinformatics. 2017;15(3):201-7. 
45. Guttman M, Rinn JL. Modular regulatory principles of large non-coding RNAs. Nature. 2012;482(7385):339-46.

46. Brantl S. Regulatory mechanisms employed by cis-encoded antisense RNAs. Curr Opin Microbiol. 2007;10(2):102-9.

47. Zhen Y, Wang F, Liang W, Liu J, Gao G, Wang Y, Xu X, Su Q, Zhang Q, Liu B. Identification of differentially expressed non-coding RNA in porcine alveolar macrophages from Tongcheng and large white pigs responded to PRRSV. Sci Rep. 2018;8(1):15621.

48. Zhang K, Ge L, Dong S, Liu Y, Wang D, Zhou C, Ma C, Wang Y, Su F, Jiang Y. Global miRNA, IncRNA, and mRNA Transcriptome profiling of endometrial epithelial cells reveals genes related to porcine reproductive failure caused by porcine reproductive and respiratory syndrome virus. Front Immunol. 2019:10:1221.

49. Zeng N, Wang C, Liu S, Miao Q, Zhou L, Ge X, Han J, Guo X, Yang H. Transcriptome analysis reveals dynamic Gene expression profiles in porcine alveolar macrophages in response to the Chinese highly pathogenic porcine reproductive and respiratory syndrome virus. Biomed Res Int. 2018; 2018:1538127

50. Sadik CD, Kim ND, Luster AD. Neutrophils cascading their way to inflammation. Trends Immunol. 2011;32(10):452-60.

51. Miller LC, Jiang Z, Sang Y, Harhay GP, Lager KM. Evolutionary characterization of pig interferon-inducible transmembrane gene family and member expression dynamics in tracheobronchial lymph nodes of pigs infected with swine respiratory disease viruses. Vet Immunol Immunopathol. 2014;159(3-4):180-91.

52. Wang X, Li C, Zhou L, Zhang N, Wang X, Ge X, Guo X, Yang H. Porcine reproductive and respiratory syndrome virus counteracts the porcine intrinsic virus restriction factors-IFITM1 and Tetherin in MARC-145 cells. Virus Res. 2014;191:92-100.

53. Yanez DC, Ross S, Crompton T. The IFITM protein family in adaptive immunity. Immunology. 2020;159(4):365-72.

54. Guo L, Niu J, Yu H, Gu W, Li R, Luo X, Huang M, Tian Z, Feng L, Wang Y. Modulation of CD163 expression by metalloprotease ADAM17 regulates porcine reproductive and respiratory syndrome virus entry. J Virol. 2014; 88(18):10448-58.

55. Johnsson P, Ackley A, Vidarsdottir L, Lui WO, Corcoran M, Grander D, Morris KV. A pseudogene long-noncoding-RNA network regulates PTEN transcription and translation in human cells. Nat Struct Mol Biol. 2013;20(4): 440-6.

56. Lee JT. Lessons from X-chromosome inactivation: long ncRNA as guides and tethers to the epigenome. Genes Dev. 2009;23(16):1831-42.

57. Tian ZJ, An TQ, Zhou YJ, Peng JM, Hu SP, Wei TC, Jiang YF, Xiao Y, Tong GZ. An attenuated live vaccine based on highly pathogenic porcine reproductive and respiratory syndrome virus (HP-PRRSV) protects piglets against HP-PRRS. Vet Microbiol. 2009;138(1-2):34-40.

58. Yang L, Xu J, Guo L, Guo T, Zhang L, Feng L, Chen H, Wang Y. Porcine Epidemic Diarrhea Virus-Induced Epidermal Growth Factor Receptor Activation Impairs the Antiviral Activity of Type I Interferon. J Virol. 2018; 92(8):e02095-17.

59. Livak KJ, Schmittgen TD. Analysis of relative gene expression data using real-time quantitative PCR and the 2(-Delta Delta C(T)) method. Methods. 2001;25(4):402-8.

60. Kim D, Langmead B, Salzberg SL. HISAT: a fast spliced aligner with low memory requirements. Nat Methods. 2015;12(4):357-60.

61. Mortazavi A, Williams BA, McCue K, Schaeffer L, Wold B. Mapping and quantifying mammalian transcriptomes by RNA-Seq. Nat Methods. 2008;5(7): $621-8$.

62. Li B, Dewey CN. RSEM: accurate transcript quantification from RNASeq data with or without a reference genome. BMC bioinformatics. 2011;12:323.

63. Robinson MD, McCarthy DJ, Smyth GK. edgeR: a bioconductor package for differential expression analysis of digital gene expression data. Bioinformatics. 2010;26(1):139-40.

64. Pertea M, Pertea GM, Antonescu CM, Chang TC, Mendell JT, Salzberg SL. StringTie enables improved reconstruction of a transcriptome from RNA-seq reads. Nat Biotechnol. 2015;33(3):290-5.

65. Trapnell C, Williams BA, Pertea G, Mortazavi A, Kwan G, van Baren MJ, Salzberg SL, Wold BJ, Pachter L. Transcript assembly and quantification by RNA-Seq reveals unannotated transcripts and isoform switching during cell differentiation. Nat Biotechnol. 2010;28(5):511-5.
66. Sun L, Luo H, Bu D, Zhao G, Yu K, Zhang C, Liu Y, Chen R, Zhao Y. Utilizing sequence intrinsic composition to classify protein-coding and long noncoding transcripts. Nucleic Acids Res. 2013;41(17):e166.

67. Kong L, Zhang Y, Ye ZQ, Liu XQ, Zhao SQ, Wei L, Gao G. CPC: assess the protein-coding potential of transcripts using sequence features and support vector machine. Nucleic Acids Res. 2007;35(Web Server issue):W345-9.

68. Anders S, Huber W. Differential expression analysis for sequence count data. Genome Biol. 2010;11(10):R106.

\section{Publisher's Note}

Springer Nature remains neutral with regard to jurisdictional claims in published maps and institutional affiliations.
Ready to submit your research? Choose BMC and benefit from:

- fast, convenient online submission

- thorough peer review by experienced researchers in your field

- rapid publication on acceptance

- support for research data, including large and complex data types

- gold Open Access which fosters wider collaboration and increased citations

- maximum visibility for your research: over $100 \mathrm{M}$ website views per year

At BMC, research is always in progress.

Learn more biomedcentral.com/submissions 